\title{
ON THE EXTENSION OF DIFFERENTIABLE FUNCTIONS
}

\section{HASSLER WHITNEY}

The author has shown previously how to extend the definition of a function of class $C^{m}$ defined in a closed set $A$ so it will be of class $C^{m}$ throughout space (see [1]). ${ }^{1}$ Here we shall prove a uniformity property: If the function and its derivatives are sufficiently small in $A$, then they may be made small throughout space. Besides being bounded, we assume that $A$ has the following property:

(P) There is a number $\omega$ such that any two points $x$ and $y$ of $A$ are joined by an arc in $A$ of length less than or equal to $\omega r_{x y}\left(r_{x y}\right.$ being the distance between $x$ and $y$ ).

This property was made use of in [2]; its necessity in the theorem is shown by two examples below.

A second theorem removes the boundedness condition in the first theorem, and weakens the hypothesis $(P)$; its proof makes use of the proof of the first theorem. We remark that in each theorem, as in [1], the extended function is a linear functional of its values in $A$.

The proof of Theorem I is obtained by examining the proof in [1]; hence we assume that the reader has this paper before him, and we shall follow its notations closely.

THEOREM I. Let $A$ be a bounded closed set in $n$-space $E$ with the property $(\mathrm{P})$, and let $m$ be a positive integer. Then there is a number $\alpha$ with the following property. Let $f(x)$ be any function of class $C^{m}$ in $A$, with derivatives $f_{k}(x)\left(\sigma_{k}=k_{1}+\cdots+k_{n} \leqq m\right)$. Suppose

$$
\left|f_{k}(x)\right|<\eta \quad\left(x \in A, \sigma_{k} \leqq m\right) .
$$

Then $f(x)$ may be extended throughout $E$ so that

$$
\left|f_{k}(x)\right|<\alpha \eta \quad\left(x \in E, \sigma_{k} \leqq m\right) .
$$

Let $d$ be the diameter of $A$, or 1 if this is larger, and let $R$ be a spherical region of radius $2 d$ with its center at a point of $A$. Set $f(x)=0$ in $E-R$. Then the extension of $f$ in $R-A$ given in [1] will be shown to have the property, using

$$
\alpha=2 n(m !)^{n}(m+1)^{3 n}\left(433 n^{1 / 2} d \omega\right)^{m} c N,
$$

where $N$ and $c$ are as given in $[1, \S \S 11,12]$. Note that $433=4 \cdot 108+1$.

Presented to the Society, September 13, 1943 ; received by the editors November 27, 1943.

1 Numbers in brackets refer to the references cited at the end of this paper. 
Set $B=A \cup(\bar{R}-R)$. We show first that for any points $x^{\prime}, x^{\prime \prime}$ of $B$,

$$
\left|R_{k}\left(x^{\prime} ; x^{\prime \prime}\right)\right|<\beta r_{x^{\prime} x^{\prime \prime} \eta}^{m-\sigma_{k}}, \quad \beta=2 n(m+1)^{n} \omega^{m} .
$$

Suppose first that $x^{\prime}$ and $x^{\prime \prime}$ are in $A$. Let $C$ be a curve in $A$ joining them, of length less than or equal to $\omega r_{x^{\prime} x^{\prime \prime}}$. The inequality is then a consequence of [2, Lemma 3]. Suppose next that one of the points is in $A$, and the other is in $\bar{R}-R$ (the case that both are in $\bar{R}-R$ is trivial). By $[1,(3.1)]$, since $r_{x^{\prime} x^{\prime \prime}} \geqq d \geqq 1$,

$$
\begin{aligned}
\left|R_{k}\left(x^{\prime} ; x^{\prime \prime}\right)\right| & \leqq \eta+\sum_{\sigma l \leqq m-\sigma_{k}} \eta r_{x^{\prime} x^{\prime \prime}}^{\sigma_{l}} \leqq r_{x^{\prime} x^{\prime} \eta \eta}^{m-\sigma_{k}}\left[1+\sum_{\sigma l m-\sigma_{k}} 1\right] \\
& \leqq(m+1)^{n} r_{x^{\prime} x^{\prime} \eta \eta}^{m-\sigma_{k}} .
\end{aligned}
$$

Now take any $x$ in $R-B$. Let $\delta^{*} / 4$ be the distance from $x$ to $B$, and let $x^{*}$ be a point of $B$ distant $\delta^{*} / 4$ from $x$. Say $x$ is in the cube $C$ of the set of cubes $K_{s}$; let $I_{\lambda_{1}}, \cdots, I_{\lambda_{t}}$ be those $I_{\lambda}$ with points in $C$ (see $[1, \S 11]$ ). Now $y^{\nu}$ is the center of $I_{\nu}$, and $x^{\nu}$ is a nearest point of $B$ to $y^{\nu}$. As noted in [1, (9.1)], $r_{y^{\nu}} x^{*}$ and $r_{y^{y}} x^{y}$ each lie between $\delta^{*} / 8$ and $\delta^{*} / 2$. Since $r_{x y^{\nu}}<\delta^{*} / 2$, we have

$$
r_{x^{\nu} x^{*}}<\delta^{*}, \quad r_{x x^{\nu}}<\delta^{*} .
$$

The definition of $\zeta$ in $[1, \S 11]$ together with $[1,(6.3)]$ gives

$$
\zeta_{\nu ; k}(x)=\psi_{k}\left(x ; x^{\nu}\right)-\psi_{k}\left(x ; x^{*}\right)=\sum_{\sigma_{l} \leqq m-\sigma_{k}} \frac{R_{k+l}\left(x^{\nu} ; x^{*}\right)}{l !}\left(x-x^{\nu}\right)^{l} .
$$

Hence

$$
\left|\zeta_{p_{i} k}(x)\right|<(m+1)^{n} \beta r_{x^{n} x^{*}}^{m-\sigma_{k}-\sigma_{l}} \boldsymbol{r}_{x x^{2} \eta}^{\sigma_{l}}<(m+1)^{n} \beta \delta^{* m-\sigma_{k}} \eta \text {. }
$$

Following $[1, \S 11]$ still, we find

$$
\left|D_{k g} g(x)-\psi_{k}\left(x ; x^{*}\right)\right|<c \sum_{\sigma_{l} \geqq m-\sigma_{k}}(m !)^{n} 2^{s \sigma_{l}} N(m+1)^{n} \beta \delta^{* m-\sigma_{k}+\sigma_{l} \eta} .
$$

As in [1], $2^{*}<108 n^{1 / 2} / \delta^{*}$; hence

$$
\left|D_{k} g(x)-\psi_{k}\left(x ; x^{*}\right)\right|<c(m !)^{n} N(m+1)^{2 n}\left(108 n^{1 / 2}\right)^{m} \beta \delta^{* m-\sigma_{k} \eta} .
$$

Moreover, since $r_{x x^{*}}<3 d,[1,(6.1)]$ gives

$$
\left|\psi_{k}\left(x ; x^{*}\right)\right|<3^{m}(m+1)^{n} d^{m} \eta .
$$

Since $\delta^{*} \leqq 4 d$ and $f(x)=g(x)$ in $R-B$, the theorem follows.

We turn now to the second theorem. We shall say $A$ satisfies $(P)$ locally if for each $x \in A$ there is a neighborhood $U$ of $x$ and a number 
$\omega$ such that any two points $y$ and $z$ of $A \cap U$ are joined by an $\operatorname{arc}$ in $A$ of length not greater than $\omega r_{x y}$.

THeOREM II. Let $A$ be a closed subset of an open set $R$ in $E$, satisfying $(P)$ locally, and let $m$ be a positive integer. Then for any continuous function $\epsilon(x)$ defined and greater than 0 in $R$ there is a continuous function $\delta(x)$ defined and greater than 0 in $A$ with the following property. Let $f(x)$ be any function of class $C^{m}$ in $A$, such that

$$
\left|f_{k}(x)\right|<\delta(x) \quad\left(x \in A, \sigma_{k} \leqq m\right) .
$$

Then $f(x)$ may be extended throughout $R$ so that

$$
\left|f_{k}(x)\right|<\epsilon(x) \quad\left(x \in R, \sigma_{k} \leqq m\right) .
$$

REMARKs. The preceding theorem is easily seen to be a consequence of this one. The present theorem holds if $E$ is replaced by a differentiable manifold $M$, in which a fixed set of coordinate systems (each one intersecting but a finite number of others) is used to measure the size of derivatives. To show this, we imbed $M$ in a Euclidean space $E^{\prime}$ (see [3, Theorem 1]), giving $A \subset R \subset R^{\prime} \subset E^{\prime}$ ( $R^{\prime}$ open in $E^{\prime}$; we let $R^{\prime}$ contain no points of the limit set of $M$ ), extend $f$ throughout $R^{\prime}$ (see the proof of [3, Lemma 4]), and consider its values in $R$.

To prove the theorem, we begin by choosing spherical regions $U_{1}, U_{2}, \cdots$, each $\bar{U}_{i} \subset R$, with the following properties:

(a) Each $U_{i}$ is in a neighborhood $U$ as described above.

(b) Each $\bar{U}_{i}$ intersects but a finite number of other $\bar{U}_{j}$.

(c) If $U_{i}$ is of radius $\rho_{i}$, and $U_{i}^{\prime}$ is the concentric region of radius $\rho_{i} / 2$, then $R=\sum U_{i}^{\prime}$.

Let $\psi^{i}(x)$ be a function of class $C^{m}$ in $E$ such that

Set

$$
\begin{array}{lr}
\psi^{i}(x)>0 & \left(x \in U_{i}^{\prime}\right), \\
\psi^{i}(x)=0 & \left(x \in E-U_{i}^{\prime}\right) .
\end{array}
$$

$$
\phi^{i}(x)=\psi^{i}(x) / \sum \psi^{i}(x) \quad(x \in R) ;
$$

then $\phi^{i}(x)$ is of class $C^{m}$ in $R$, and

$$
\begin{array}{rlr}
\phi^{i}(x) & =0 & \left(x \in R-U_{i}^{\prime}\right), \\
\sum \phi^{i}(x) & =1 & (x \in R) .
\end{array}
$$

The extension of $f(x)$ is defined as follows. Set

$$
\begin{array}{lr}
f^{i}(x)=\phi^{i}(x) f(x) & (x \in A), \\
f^{i}(x)=0 & \left(x \in R-U_{i}\right) .
\end{array}
$$

Then $f^{i}$ is of class $C^{m}$ in $A \cup\left(R-U_{i}\right)$. Extend it as in [1] (using a fixed 
subdivision of $U_{i}-A$; we could set $f^{i}(x)=0$ in $\left.E-R\right)$ to be of class $C^{m}$ in $R$ (or $E$ ). (Note that if $A \cap U_{i}^{\prime}=0$, then $f^{i}(x)=0, x \in R$.) Set

$$
f(x)=\sum f^{i}(x) \quad(x \in R) .
$$

Then $f$ is an extension of class $C^{m}$ of its values in $A$. We must show that it satisfies the condition of smallness.

Choose $a_{i} \geqq 1$ so that

$$
\left|\phi_{k}^{i}(x)\right| \leqq a_{i} \quad\left(x \in R, \sigma_{k} \leqq m\right),
$$

then if $\left|f_{k}(x)\right|<\eta\left(x \in A \cap U_{i}^{\prime}\right)$,

$$
\left|f_{k}^{i}(x)\right|=\left|\sum_{\sigma l \leqq \sigma_{k}} \phi_{l}^{i}(x) f_{k-l}(x)\right| \leqq(m+1)^{n} a_{i} \eta \quad(x \in A) .
$$

By the choice of $U_{i}$, there is an $\omega_{i}$ such that any $x^{\prime}$ and $x^{\prime \prime}$ in $A \cap U_{i}$ are joined by an arc in $A$ of length not greater than $\omega_{i} r_{x^{\prime} x^{\prime \prime}}$. Set $\sigma_{i}=\max \left(1,2 / \rho_{i}\right)$. If $R_{k}^{i}$ is the remainder for $f_{k}^{i}$, we shall show that for any $x^{\prime}$ and $x^{\prime \prime}$ in $A \cup\left(R-U_{i}\right)$,

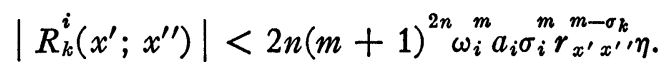

If $x^{\prime}$ and $x^{\prime \prime}$ are both in $U_{i}$, we apply [2, Lemma 3]. If $x^{\prime} \in R-U_{i}$ and $x^{\prime \prime} \in U_{i}^{\prime}$, or vice versa, then $r_{x^{\prime} x^{\prime \prime}} \geqq \rho_{i} / 2$, and the proof in the preceding theorem applies; we consider separately the cases $\rho_{i} / 2 \geqq 1$, $\rho_{i} / 2<1$, using $r_{x^{\prime} x^{\prime \prime}} \geqq 1$ and $\sigma_{i} r_{x^{\prime} x^{\prime \prime}} \geqq 1$ respectively. If $x^{\prime} \in R-U_{i}$ and $x^{\prime \prime} \in R-U_{i}^{\prime}$, or vice versa, $R_{k}^{i}=0$, since $\phi_{l}^{i}\left(x^{\prime}\right)=\phi_{l}^{i}\left(x^{\prime \prime}\right)=0$. The proof of the preceding theorem now shows that for some $\alpha_{i}$, if

then

$$
\left|f_{k}\left(x^{\prime}\right)\right|<\eta \quad\left(x^{\prime} \in A \cap U_{i}^{\prime}, \sigma_{k} \leqq m\right),
$$

$$
\left|f_{k}^{i}(x)\right|<\alpha_{i} \eta \quad\left(x \in R, \sigma_{k} \leqq m\right) .
$$

(We may set $\alpha_{i}=1$ if $A \cap U_{i}^{\prime}=0$.)

Given $\epsilon(x)$, we determine $\delta(x)$ as follows. For each $x \in R$ there is a set of numbers $\lambda_{1}, \cdots, \lambda_{s}, s=s(x)$, such that

$$
x \in \text { each } U_{\lambda_{j}}^{\prime}, \quad x \in \text { no other } U_{l}^{\prime} .
$$

Because of (b), $s$ is finite. Set $\alpha(x)=\alpha_{\lambda_{1}}{ }^{3}+\cdots+\alpha_{\lambda_{\text {}}}$.

We can clearly choose a continuous function $\beta(x)$ in $R$ such that

$$
\alpha(x)<\beta(x) \quad(x \in R) .
$$

We may now choose a continuous function $\delta\left(x^{\prime}\right)>0$ in $A$ such that 
for any $x^{\prime} \in A$, if $U_{\mu_{1}}^{\prime}, \cdots, U_{\mu_{t}}^{\prime}$ are the $U_{j}^{\prime}$ containing $x^{\prime}$, then

$$
\delta\left(x^{\prime}\right) \leqq \epsilon(x) / \beta(x) \quad\left(x \in U_{\mu_{1}}^{\prime} \cup \ldots \cup U_{\mu_{t}}^{\prime}\right) .
$$

Now take any $f$ of class $C^{m}$ in $A$, with $\left|f_{k}(x)\right|<\delta(x)\left(x \in A, \sigma_{k} \leqq m\right)$; the extension of $f$ through $R$ has been defined. Take any $x \in R$; define $\lambda_{1}, \cdots, \lambda_{s}$ as above. Then

$$
\left|f_{k}\left(x^{\prime}\right)\right|<\delta\left(x^{\prime}\right) \leqq \epsilon(x) / \beta(x) \quad\left(x^{\prime} \in A \cap U_{\lambda_{j}}^{\prime}, \sigma_{k} \leqq m\right),
$$

and hence

$$
\left|f_{k}^{\lambda_{j}}(x)\right|<\alpha_{\lambda_{j}} \epsilon(x) / \beta(x) \quad\left(\sigma_{k} \leqq m\right) .
$$

Since $f_{k}(x)=f_{k}^{\lambda_{1}}(x)+\cdots+f_{k}^{\lambda_{s}}(x)$,

$$
\left|f_{k}(x)\right|<\alpha(x) \epsilon(x) / \beta(x)<\epsilon(x)
$$

for $\sigma_{k} \leqq m$, which completes the proof.

EXAMPLES. (1) Let $A$ consist of a point, together with a sequence of points approaching it. Letting $f(x)=1$ at a finite number of points of the sequence, and $f(x)=0$ in the rest of $A$ shows (with $m=1$ ) that the theorem fails here.

(2) Let $A$ be the closed region of the plane defined by (a) $x^{2}+y^{2} \leqq 1$, and (b) either $x \leqq 0$ or $|y| \geqq x^{8 / 2}$. Let $f(x, y)=0$ if $x \leqq 0$, and set

$$
f(x, y)=\left\{\begin{aligned}
\gamma x^{2} /\left(1+\gamma^{2} x^{2}\right) & \text { if } \quad x \geqq 0, y>0, \\
-\gamma x^{2} /\left(1+\gamma^{2} x^{2}\right) & \text { if } \quad x \geqq 0, y<0 .
\end{aligned}\right.
$$

We see easily that $f$ is of class $C^{1}$ in $A$. (It would not be if, in (b), we used $|y| \geqq x^{2}$.) The maximum $\partial f / \partial x$ occurs at $x=1 /\left(3^{1 / 2} \gamma\right)$, and has the value $9 /\left(8 \cdot 3^{1 / 2}\right)$. Set

$$
p=\left(1 / 3^{1 / 2} \gamma, 1 / 3^{3 / 4} \gamma^{3 / 2}\right), \quad q=\left(1 / 3^{1 / 2} \gamma,-1 / 3^{3 / 4} \gamma^{3 / 2}\right)
$$

Then

$$
\frac{f(p)-f(q)}{r_{p q}}=\frac{2 \gamma / 3 \gamma^{2}}{1+\gamma^{2} / 3 \gamma^{2}} \div \frac{2}{3^{3 / 4} \gamma^{3 / 2}}=\frac{3^{3 / 4}}{4} \gamma^{1 / 2} .
$$

Hence, in any extension of $f$ through the plane, we must have $|\partial f / \partial y| \geqq 3^{3 / 4} \gamma^{1 / 2} / 4$ at some point (between $p$ and $q$ ); yet $|f|,|\partial f / \partial x|$ and $|\partial f / \partial y|$ are uniformly bounded for all $\gamma>1$. Taking $\gamma$ arbitrarily large shows that the conclusion of the theorem does not hold.

\section{REFERENCES}

1. H. Whitney, Analytic extensions of differentiable functions defined in closed sets, Trans. Amer. Math. Soc. vol. 36 (1934) pp. 63-89. 
2. - Functions differentiable on the boundaries of regions, Ann. of Math. vol. 35 (1934) pp. 482-485.

3. — Differentiable manifolds, Ann. of Math. vol. 37 (1936) pp. 645-680.

4. - Differentiable functions defined in arbitrary subsets of Euclidean space, Trans. Amer. Math. Soc. vol. 40 (1936) pp. 309-317. Further references are given here.

5. H. O. Hirschfeld, Continuation of differentiable functions through the plane, Quart. J. Math. Oxford Ser. vol. 7 (1936) pp. 1-15.

6. M. R. Hestenes, Extension of the range of a differentiable function, Duke Math. J. vol. 8 (1941) pp. 183-192.

HARVARD UNIVERSITY

\section{THE SYMMETRIC JOIN OF A COMPLEX}

\section{E. CLARK}

1. The definition of $J$. Let $K$ be a polyhedron. With each pair of distinct points $p, q$ of $K$ we associate a closed line segment $p q$. No distinction is made between $p$ and $q$ and the corresponding end points of $p q$. The length of $p q$ is a continuous function of $p$ and $q$, and the length approaches zero if $p$ and $q$ approach a common limit. Distinct segments do not intersect except at a common end point. The points of these segments with their obvious natural topology make up $J$, the symmetric join of $K$. This space arises in [4] ${ }^{1}$ in connection with the problem of finding the chords of a manifold that are orthogonal to the manifold.

2. The subdivision of $J$. Let the mid-point of $p q$ be denoted by $\Lambda p \times q=\Lambda q \times p$, and let $p=\Lambda p \times p$. These points $\Lambda p \times q$ make up the symmetric product $S$ of $K$. Let the mid-point of the segment from $p$ to $\Lambda p \times q$ be denoted by $p \times q$, and let $p=p \times p$. These points $p \times q$ make up the topological product $P=K \times K$. Consider the closed segment of $p q$ from $p \times q$ to $q \times p$, it being understood that this segment is the point $p$ when $p=q$. All such segments form the "neighborhood" $N_{S}$. Clearly $N_{S}$ can be homotopically deformed in $N_{S}$ along the segments $p q$ upon $S$ with $S$ remaining pointwise invariant. Finally consider the closed segment of $p q$ from $p$ to $p \times q$, it being understood that this segment is the point $p$ when $p=q$. All such segments form the "neighborhood" $N_{K}$. Clearly $N_{K}$ can be homotopically deformed in $N_{K}$ along the segments $p q$ upon $K$ with $K$ remaining pointwise invariant.

Received by the editors July $23,1943$.

${ }^{1}$ Numbers in brackets refer to the References at the end of the paper. 